\title{
L'acide linoléique conjugué en nutrition porcine
}

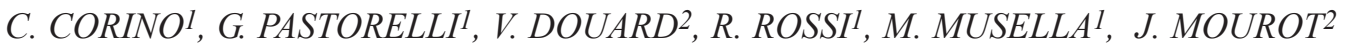 \\ ${ }^{1}$ Facoltà di Medicina Veterinaria, Dipartimento di Scienze e Tecnologie Veterinarie per la Sicurezza Alimentare, \\ Via Celoria, 10-20133 Milan, Italie \\ ${ }^{2}$ INRA, Unité Mixte de Recherches Système d'Elevage, Alimentation Animale et Humaine, F-35590 Saint-Gilles \\ Courriel : Jacques.Mourot@rennes.inra.fr
}

$\mathrm{Au}$ cours des vingt dernières années, on a mis en évidence des propriétés intéressantes de certains isomères des CLA chez de nombreux modèles animaux et montré leur intérêt nutritionnel chez l'homme. La tendance en élevage est donc d'augmenter la teneur en CLA des produits animaux en jouant sur l'alimentation de l'animal. Toutefois, l'innocuité de toutes les formes des CLA n'est pas démontrée et une prudence est encore conseillée dans ces nouvelles orientations en élevage.

L'acide linoléique conjugué ou «Conjugated Linoleic Acid» (CLA) est un terme générique qui désigne un mélange complexe d'isomères géométriques et de positions de l'acide linoléique possédant deux doubles liaisons conjuguées. Ces doubles liaisons de configuration cis-trans, trans-cis, transtrans ou cis-cis peuvent se situer en différentes positions de la chaîne carbonée.

Ces CLA, présents à l'état naturel dans certains produits animaux, comme la matière grasse de la viande des ruminants et les produits laitiers (Chin et al 1992, Chilliard et al 2001), peuvent présenter des propriétés intéressantes vis-à-vis de l'animal mais aussi $\mathrm{du}$ consommateur. Ils auraient des effets sur la santé humaine en réduisant la fréquence des maladies cardio-vasculaires et de certains cancers (Jahreis et al 2000, Gnadig et al 2001, Azain 2003, O'Shea et al 2004, Pariza 2004, Wang et al 2004). De ce fait, ils suscitent un intérêt certain et posent des questions quant aux recommandations alimentaires actuelles.

Contrairement aux ruminants, les animaux monogastriques n'ont pas la capacité de synthétiser les CLA. Ceux présents dans les tissus viennent directement de l'alimentation. Des essais ont été réalisés pour préciser les conséquences de l'introduction des CLA dans l'aliment sur les performances de croissance et la santé des animaux, et pour essayer d'augmenter leur teneur dans la viande, sachant qu'il existe une relation directe entre les lipides ingérés chez les animaux monogastriques et les acides gras déposés (Mourot et Hermier 2001). La mise à disposition sur le marché de CLA et l'intérêt suscité par un certain nombre de grands groupes de l'alimentation animale laissent entrevoir un développement de cette pratique en élevage porcin. Un premier bilan de ces travaux est présenté dans cette synthèse.

\section{1 / Effets sur les performan- ces de croissance et la com- position corporelle}

Les CLA utilisés en nutrition animale sont des mélanges synthétiques, contenant principalement deux isomères, l'isomère $\mathrm{c} 9, \mathrm{t} 11-\mathrm{CLA}$ et 1'isomère t10,c12-CLA, mais aussi, et selon les sources commerciales, des isomères 8,10 et 11,13 .

Selon les modes de synthèse des CLA, le rapport des isomères c9,t11-CLA et t10,c12-CLA peut varier. Le contenu en isomères des produits commerciaux est compris entre $65-78 \%$, avec un rapport t10,c12-CLA/c9,t11-CLA en moyenne de 1,04 mais aussi avec des valeurs de 1,44 (Yu et al 2003).

\section{1 / Effets sur la mise en place des tissus}

Chez le porc, les teneurs en acides gras des aliments de gestation et de lactation peuvent modifier l'adiposité des

Figure 1. Consommation journalière de CLA et concentration en CLA dans le muscle, le tissu adipeux du porc et le colostrum de truie.

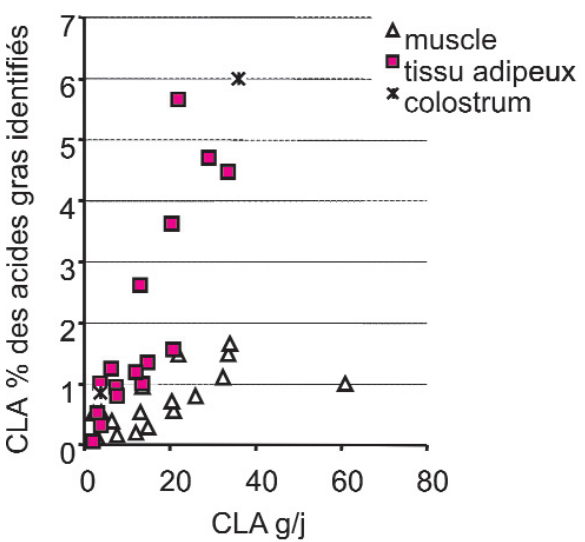

Colostrum : Bee (2000), Bontempo et al (2004).

Muscle : Bee (2001), Eggert et al (2001), Gatlin et al (2002), Joo et al (2002), Migdal et al (2004), Ostrowska et al (2003), Thiel-Cooper et al (2001), Tischendorf et al (2002), Wiegand et al (2002) La consommation alimentaire est supposée égale à $2,7 \mathrm{~kg} / \mathrm{j}$. 
porcelets à la naissance et au sevrage. Chez les truies en phase de gestationlactation recevant des CLA alimentaires, des CLA sont retrouvés dans le colostrum et le lait (figure 1). Ainsi comme certains autres acides gras, les CLA pourront jouer un rôle éventuel sur la mise en place des tissus adipeux.

A taux de lipides constants, la présence de CLA dans les régimes maternels au cours de la gestation n'influence pas la teneur en lipides et le nombre des cellules chez le porcelet à la naissance (Douard et al 2004a). Par contre, au cours de la lactation un régime enrichi en CLA entraîne une diminution significative du nombre total de cellules (préadipocytes + adipocytes), comparativement à un régime riche en acides gras essentiellement saturés ou monoinsaturés. La teneur totale en lipides de la carcasse est également plus faible chez les porcelets élevés par des mères ayant consommé des CLA pendant la lactation (Douard et al 2004a).

\section{2 / Effets sur la croissance}

Les effets des CLA sur les performances de croissance en élevage porcin ont fait l'objet de synthèses récentes (Azain 2003, Dugan et al 2004). Les références des principaux travaux réalisés sur l'utilisation des CLA en alimentation porcine (nombre de sujets, poids initial et final, $\%$ de produit CLA utilisé et teneur en CLA) sont rapportés dans le tableau 1. Dans l'ensemble, ces travaux se sont intéressés aux porcs en croissance entre 5,6 et $172 \mathrm{~kg}$ PV. La variabilité entre les études est importante à la fois en terme de consommation avec une distribution moyenne de CLA de 13,4 $\pm 9,7 \mathrm{~g} / \mathrm{j}$ et en terme de durée d'expérimentation (72,1 \pm $22,4 \mathrm{j}$ ).
L'apport de CLA dans l'alimentation ne semble pas modifier le gain moyen quotidien. La consommation alimentaire, en condition d'alimentation à volonté est réduite, ce qui induit dans la majorité des observations, une amélioration de l'efficacité alimentaire chez les porcs recevant les régimes avec CLA. Pour un ensemble d'études, la réduction moyenne de l'indice de consommation est de 5,7 $\pm 3,6 \%$ (Bee 2001, Corino et al 2003, Eggert et al 2001, Migdal et al 2004, Ostrowska et al 2003, Waylan et al 2002, Wiegand et al 2002).

Cependant, il n'est pas possible de mettre en évidence un effet de la dose ou de la durée du traitement. Ainsi, avec un apport de $1,25 \mathrm{~g}$ de CLA $/ \mathrm{kg}$ d'aliment, la diminution de l'indice de consommation est de 5,5\% (Ostrowska et al 1999) et pour $20 \mathrm{~g} / \mathrm{kg}$, elle est de $4,7 \%$ (Bee 2001). Pour une durée de

Tableau 1. Références bibliographiques des principaux travaux concernant l'utilisation des CLA en nutrition porcine.

\begin{tabular}{|c|c|c|c|c|c|c|}
\hline & \multirow[t]{2}{*}{$\begin{array}{c}\text { Porc } \\
\mathrm{n} .\end{array}$} & \multicolumn{2}{|c|}{$\begin{array}{c}\text { Poids } \\
\text { kg }\end{array}$} & \multirow{2}{*}{$\begin{array}{c}\text { Durée } \\
\mathbf{j}\end{array}$} & \multirow{2}{*}{$\begin{array}{c}\text { CLA } \\
\text { dans le régime } \\
\%\end{array}$} & \multirow{2}{*}{$\begin{array}{c}\text { CLA } \\
\%\end{array}$} \\
\hline & & initial & final & & & \\
\hline \multicolumn{7}{|l|}{ Porc en croissance } \\
\hline King et al (2004) & 6 & 5,6 & 25,6 & 35 & 1,5 & 60 \\
\hline Demaree et al (2002) & 6 & 5,6 & 26 & 35 & 3 & 60 \\
\hline Smith et al (2002) & 6 & 5,6 & 25,6 & 35 & 1,5 & 60 \\
\hline Bee (2000) & 10 & 9,8 & 22 & 35 & 2 & 59 \\
\hline Ramsay et al (2001) & 7 & 20 & 55 & 48 & $0,250,512$ & 67 \\
\hline Tischendorf et al (2002) & 40 & 23,5 & 114 & 131 & 2 & 54 \\
\hline Thiel Cooper et al (2001) & 8 & 26,3 & 116 & 90 & $0,20,420,831,67$ & 60 \\
\hline Wiegand et al (2002) & 23 & 28 & 115 & 95 & 1,25 & 60 \\
\hline Dugan et al (2001) (2003) & 36 & 36 & 115 & 83 & $0,250,5$ & 65 \\
\hline O'Quinn et al (2000) & 20 & 33,4 & 118,7 & 85 & $0,250,51$ & 69 \\
\hline Waylan et al (2002) & 36 & 45,5 & 114,6 & 60 & 0,5 & 66 \\
\hline Gatlin et al (2002) & 24 & 72 & 113 & 47 & 1 & 60 \\
\hline Ostrowska et al (1999) & 10 & 56,6 & 107 & 56 & $0,1250,250,50,751$ & 55 \\
\hline Ostrowska et al (2003) & 5 & 57,2 & 106,2 & 56 & $0,1250,250,50,751$ & 55 \\
\hline D'Souza et al (2002) & 72 & 60 & 105 & 42 & 0,5 & $?$ \\
\hline Dugan et al (1997) (1999) & 54 & 61,5 & 106 & 45 & 2 & 50 \\
\hline Dunshea et al (2002) & 80 & 62 & 110 & 49 & 0.4 & 55 \\
\hline Bee (2001) & 8 & 70 & 105 & 35 & 2 & 58,9 \\
\hline Migdal et al (2004) & 20 & 70 & 130 & 80 & 2 & 60 \\
\hline Eggert et al (2001) & 10 & 75 & 120 & 56 & 1 & 60 \\
\hline Joo et al (2002) & 5 & 77 & 105 & 28 & 12,55 & 91 \\
\hline Corino et al (2003) & 12 & 97 & 172 & 101 & $0,250,5$ & 65 \\
\hline Corino et al (données non publiées) & 18 & 104 & 155 & 82 & 0,75 & 50 \\
\hline \multicolumn{7}{|l|}{ Truie et Immunité } \\
\hline Hontecillas et al (2002) & 16 & $4.8-5,5$ & - & 49 & 2,21 & 60 \\
\hline Bassaganya-Riera et al (2001a) & 8 & 5,3 & $34-44$ & 49 & $0,671,332$ & 60 \\
\hline Weber et al (2001) & 48 & 7,6 & 42,3 & 63 & 1 & 60 \\
\hline Corino et al (2002) & 14 & 12,4 & 26 & 28 & 0,51 & 65 \\
\hline Bee (2000) & 6 & \multicolumn{3}{|c|}{ Truie : 90j G+35j L } & 2 & 60 \\
\hline Bontempo et al (2004) & 8 & \multicolumn{3}{|c|}{ Truie : 8j G+21 L } & 0,5 & 50 \\
\hline Corino et al (données non publiées) & 7 & \multicolumn{3}{|c|}{ Truie : 7j G+21j L } & 0,5 & 50 \\
\hline
\end{tabular}


distribution de 35 jours, la diminution est de 4,7\% (Bee 2001), pour 131 jours elle est de 0,3\% (Tischendorf et al 2002). Le poids de l'animal semble aussi un facteur à prendre en considération, puisque la meilleure augmentation de l'efficacité alimentaire est observée chez les animaux lourds abattus à plus de $160 \mathrm{~kg}$ avec $+12,5 \%$ après une période de 101 jours de distribution (Corino et al 2003).

\section{3 / Effets sur la composition corporelle}

Les effets des CLA sur la composition corporelle et leur mécanisme d'action ont fait 1'objet de synthèses (Mersmann 2002, Pariza 2004, Wang et Jones 2004). Comme pour les paramètres de croissance, la variabilité entre les quantités de CLA distribuées et la durée des traitements est importante. Globalement, l'ensemble des études semble montrer une réduction de l'adiposité (Bee 2001, Corino et al 2003, Dugan et al 1997, Eggert et al 2001, Gatlin et al 2002, Migdal et al 2004, Ostrowska et al 1999, 2003).

Cependant, il n'est pas observé de relation entre la dose ingérée et/ou le temps de distribution du régime sur la réduction de l'adiposité de la carcasse. Ainsi, 1,25 g de CLA/kg aliment entraîne une diminution de l'adiposité de 12,6\% (Ostrowska et al 1999) alors que pour $20 \mathrm{~g}$ de CLA, elle est de 7,3\% (Bee 2001). Pour une durée de 35 j, elle est de 7,3\% (Bee 2001) et pour $101 \mathrm{j}$ de distribution elle est de 8,8\% (Corino et al 2003). La réduction de la masse adipeuse peut aussi entraîner une augmentation de la teneur en viande maigre de la carcasse (TVM) ce qui peut présenter un intérêt économique pour l'éleveur, ce dernier étant rémunéré, en France, sur la valeur de ce critère.

La découpe de la carcasse montre, chez les animaux recevant des CLA, que le poids des morceaux contenant essentiellement des tissus maigres (longe, jambon) est augmenté alors que le poids des morceaux riches (bardière, poitrine) en tissus gras est diminué (tableau 2).

\section{4 / Effet sur le métabolisme des lipides}

En plus d'une diminution des poids des tissus adipeux, certains auteurs mettent en évidence une baisse des lipides totaux dans ces tissus adipeux (Ostrowska et al 1999, Douard et al 2004b), sans réduction dans le tissu musculaire (Douard et al 2004b). L'effet sur la réduction de l'adiposité est principalement attribué à l'isomère t10,c12-CLA. Chez le porc, le mode d'action des CLA pour diminuer l'adiposité serait la conséquence de plusieurs facteurs :

- une réduction de la lipogenèse due à une diminution de l'activité de l'acétyl-CoA-carboxylase, enzyme clé de la lipogenèse (Corino et al 2003) ;

- une augmentation de la lipolyse (Ostrowska et al 2002) et de l'oxydation des acides gras qui peut être mise en relation avec l'augmentation de la créatine-palmitoyl-transférase (Park et al 1997, Martin et al 2000). Chez la souris, le traitement avec les CLA induit une augmentation du métabolisme énergétique (West et al 2000), mais le même effet n'est pas observé chez le porc et la truie (Muller et al 2000). En accord avec ce résultat, on peut observer une réduction du quotient respiratoire chez le rat (West et al 1998) mais pas chez le porc ou la truie (Muller et al 2000) ;

- une réduction ou un retard de la différenciation des préadipocytes est observé en culture in vitro pour les préadipocytes de porcelets issus de mères ayant consommé un régime enrichi en CLA. Ces préadipocytes vont se différencier en adipocytes dont le diamètre sera plus petit que ceux obtenus chez des animaux n'ayant pas reçu de CLA (Douard résultats non publiés). D'autres études ont également montré une réduction du niveau d'expression de PPAR $\gamma$ (Kang et al 2003) ainsi qu'une augmentation de l'apoptose et une réduction de la prolifération des préadipocytes (Corino et al 2005).

A ces différents facteurs, on peut ajouter une réduction de la consommation d'énergie qui cependant n'est pas toujours observée et qui ne justifierait pas à elle seule l'importance de la réduction de l'adiposité de la carcasse.

Tableau 2. Effet des CLA dans le régime (apport 0,7\%) entre 35 et $105 \mathrm{~kg}$ de poids vif sur les principaux poids des morceaux de découpe (expression en \% du poids de la carcasse) (Mourot données non publiées).

\begin{tabular}{|c|c|c|c|c|c|c|c|c|}
\hline & $\begin{array}{c}\text { Poids } \\
\text { carcasse } \\
\mathbf{( k g )}\end{array}$ & TVM & Jambon & Longe & Bardière & Poitrine & Epaule & Panne \\
\hline Témoin & 86,3 & 59,2 & 22,68 & 25,36 & 7,24 & 13,11 & 23,19 & 1,48 \\
CLA & 86,1 & 60,5 & 23,53 & 26,50 & 5,99 & 12,10 & 22,39 & 1,31 \\
\hline RSD & 3,21 & 2,05 & 0,90 & 1,13 & 0,91 & 0,76 & 0,94 & 0,26 \\
Effet & NS & $\mathrm{P}<0,02$ & $\mathrm{P}<0,05$ & $\mathrm{P}<0,06$ & $\mathrm{P}<0,01$ & $\mathrm{P}<0,03$ & $\mathrm{P}<0,04$ & $\mathrm{NS}$ \\
\hline
\end{tabular}

$n=18$ porcs par lot.
Chez le porc lourd, un effet des CLA sur la taille des cellules a été observé (Corino et al 2005). L'apport de 0,75\% de CLA dans l'alimentation diminue la taille des adipocytes dans le tissu adipeux sous-cutané dorsal en raison d'une diminution de l'accumulation des lipides. De plus, dans le tissu adipeux de porcs alimentés avec des CLA le taux de prolifération des préadipocytes était inférieur à celui du contrôle. Il existe peu de données publiées sur ce sujet in vivo. Des études en cours sur le porcelet (Douard et Mourot données non publiées) vont dans le sens des observations in vitro (Azain et al 2003) montrant une diminution de la prolifération de cellules 3T3-L1, une lignée de cellules généralement utilisée pour l'étude du développement des adipocytes.

En conclusion, on peut retenir une diminution de l'adiposité chez le porc qui très réduite en comparaison des effets observés chez les animaux de laboratoire : Park et al (1997) ont observé chez la souris recevant des CLA une diminution de près de $50 \%$ des lipides corporels.

\section{2 / Effets sur la qualité de la viande}

Les qualités technologiques et sensorielles de la viande ne semblent pas modifiées, ou bien les effets sont très limités chez les animaux recevant des CLA alimentaires par rapport à une alimentation standard (tableau 3). Une évaluation subjective de la couleur de la viande ne montre aucune différence et les indices de couleur de la longe et du jambon ne sont pas affectés par le traitement.

La valeur $\mathrm{du} \mathrm{pH}$ post-mortem des muscles longissimus et semimembranosus n'est pas affectée par le traitement,

\section{1 / Qualités technologiques de la viande}


Tableau 3. Influence des CLA sur les paramètres de qualité de la viande.

\begin{tabular}{|c|c|c|}
\hline $\begin{array}{c}\text { Paramètres de qualité } \\
\text { de la viande }\end{array}$ & Effet & Références bibliographiques \\
\hline $\begin{array}{l}\text { Couleur } \\
\text { - évaluation subjective } \\
\text { - } L^{*}, a^{*}, b^{*}\end{array}$ & $\begin{array}{l}\text { - Influence NS } \\
\text { - Influence NS } \\
-\uparrow b^{*} \\
-\uparrow L^{*}\end{array}$ & $\begin{array}{l}5,6,7,8,10,12,15,16,17 \\
1,2,4,6,10,12,14,15 \\
3,5,11,16,17 \\
11,16\end{array}$ \\
\hline Glycogène & - Influence NS & 5,6 \\
\hline $\mathrm{pH}$ & $\begin{array}{l}- \text { Influence NS } \\
-\uparrow \\
-\downarrow\end{array}$ & $\begin{array}{l}2,6,8,10,14,16,17 \\
4,7 \\
3,5,11,13\end{array}$ \\
\hline Perte en eau & - Influence NS & $1,5,6,7,8,10,11,12,14,15$ \\
\hline Force de cisaillement & - Influence NS & $5,6,14$ \\
\hline IMF & $\begin{array}{l}- \text { Influence NS } \\
-\uparrow\end{array}$ & $\begin{array}{l}1,2,3,4,14 \\
5,6,9,10,11,13,16,17\end{array}$ \\
\hline Analyse sensorielle & - Influence NS & $2,5,6,11,14,15,16,17$ \\
\hline
\end{tabular}

1 Bee (2001), 2 Corino et al (2003), 3 Corino et al (données non pubbliées), 4 D'Souza et Mullan (2002), 5 Dugan et al (1999), 6 Dugan et al (2003), 7 Dunshea et al (2002), 8 Eggert et al (2001), 9 Gatlin et al (2002), $10 \mathrm{Joo}$ et al (2002), 11 Migdal et al (2004), 12 O'Quinn et al (2000), 13 ThielCooper et al (2001), 14 Tischendorf et al (2002), 15 Waylan et al (2002), 16 Wiegand et al (2001), 17 Wiegand et al (2002).

des auteurs ayant toutefois rapporté de faibles variations en augmentation ou en diminution. Globalement, on peut donc conclure à une absence d'effet, ces résultats étant conformes à de nombreux travaux ne montrant pas d'influence directe des acides gras alimentaires sur la valeur du $\mathrm{pH}$ (Lebret et al 1999). Aucune différence n'est observée sur la teneur en glycogène du muscle.

Les pertes exsudatives ne sont pas affectées par le traitement avec les CLA. Il en est de même pour la force de cisaillement chez le porc, alors que des effets significatifs ont été observés sur les variations du contenu en collagène dans le muscle du lapin (Corino et al 2004).

Les variations de l'indice de peroxydation des acides gras (indice TBA) montrent un effet significatif en faveur des animaux recevant la dose la plus élevée de CLA, mais uniquement lorsque le temps d'oxydation est élevé (Corino et al 2003). Globalement, il apparaît donc que l'addition de CLA n'a pas un effet important sur l'oxydation des lipides dans la viande.

\section{2 / Qualité nutritionnelle}

Si l'on considère que les CLA ont un intérêt nutritionnel chez l'homme, la valeur nutritionnelle des viandes de porc recevant des CLA alimentaires est augmentée. En effet, les CLA ingérés sont retrouvés dans les tissus adipeux et musculaires (figure 1) montrant une nouvelle fois l'effet de la nature des lipides sur les acides gras déposés (Lebret et Mourot 1998).

L'efficacité du passage aliment-tissu est différente selon les isomères. Ostrowska et al (2003a), reportent pour le c9,t11-CLA une efficacité moyenne de $46,4 \%$ dans la graisse sous-cutanée mation alimentaire est supposée égale à $2,7 \mathrm{~kg} / \mathrm{j}$.

CTR : animaux témoins. et al (2002), (10) Wiegand et al (2002). et de $0,64 \%$ dans la graisse intramusculaire et pour le t10,c12-CLA une efficacité moyenne seulement de 13,3 et de $0,31 \%$ respectivement.

Chez les porcs recevant les CLA, la teneur en acides gras saturés (AGS) est augmentée de 9,3 points $(+24,6 \%$ $\mathrm{P}<0,001$ ), celle en acides gras monoinsaturés (AGMS) est diminuée de 8,2 points $(-18,5 \quad \% \quad \mathrm{P}<0,001)$ (figure 2). Les variations des acides gras polyinsaturés (AGPI) ne sont pas concordantes entre les différentes études. Cela serait à mettre en relation avec les différents modes d'introduction des CLA dans la ration (en substitution de lipides de différentes compositions en acides gras) et aussi en fonction de l'adiposité de la carcasse au moment de l'introduction dans le régime. L'augmentation des AGPI (acides gras quasi exclusivement alimentaires) chez les animaux plus maigres peut s'expliquer par le fait que les lipides déposés contiennent une part moins importante de lipides d'origine endogène que les porcs plus gras.

La diminution des AGMS est principalement due à la variation de l'acide oléique (Douard et al 2004b), les CLA pouvant induire une diminution de l'expression du gène de la stéaroyl CoA désaturase (Lee et al 1998, Smith et al 2002). Ceci expliquerait indirectement l'augmentation de la teneur en AGS qui concerne surtout le C16:0, produit terminal de la synthèse des acides gras. L'augmentation en C16:1 serait au

Figure 2. Influence des CLA sur la composition en acides gras du tissu adipeux de couverture (acides gras saturés, AGS, et acides gras monoinsaturés, AGMS).

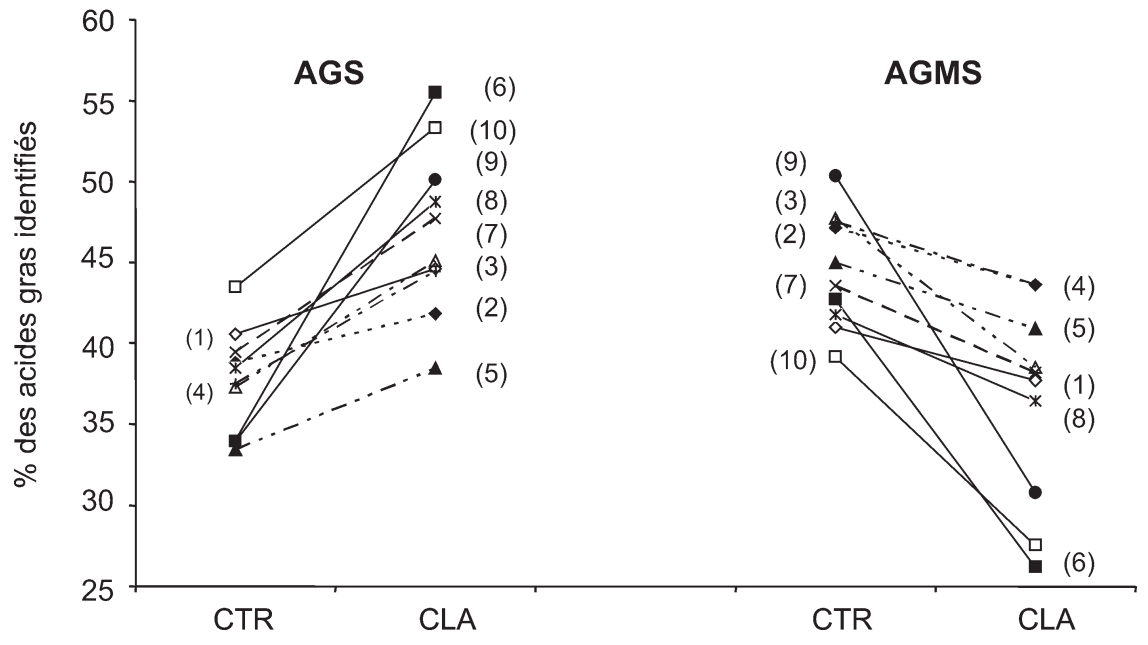

Tissu adipeux : Bee (2001), Eggert et al (2001), Corino et al (2003), Gatlin et al (2002), Kramer et al (2004), Ostrowska et al (2003), Thiel-Cooper et al (2001), Wiegand et al (2002). - La consom-

(1) Bee (2001), (2) Corino et al (2003), (3) Dugan et al (2003), (4) Eggert et al (2001), (5) Gatlin et al (2002), (6) King et al (2004), (7) O'Quinn et al (2000), (8) Smith et al (2002), (9) Tischendorf 
contraire à mettre en relation avec la réduction de l'adiposité des carcasses et due à un effet de concentration (Ostrowska et al 2003).

La fermeté du tissu adipeux est également accrue grâce à cette augmentation des acides gras saturés, la consistance des tissus adipeux étant étroitement corrélée avec le point de fusion des graisses (Wood et al 1978). Ainsi l'indice d'iode est réduit de 7-9 \% chez le porc lourd (Corino et al 2003) et de $14 \%$ chez le porc abattu à $80 \mathrm{~kg}$ de poids vif. Cela peut être déterminant pour obtenir des produits présentant une bonne aptitude à la transformation.

Les CLA modifient également la distribution des acides gras saturés sur la structure des triglycérides dans le tissu adipeux du porc entraînant un contenu élevé en acides gras saturés en position $1-3$, ce qui serait associé à une augmentation du point de fusion des lipides (King et al 2004).

\section{3 / Effets sur la réponse immunitaire}

Les propriétés immunomodulatoires des CLA ont fait l'objet d'une synthèse récente de O'Shea et al (2004). L'activité des CLA s'explique par :

- une activité d'immunomodulation qui se traduit par une augmentation de la teneur en $\operatorname{IgG}, \operatorname{Ig}$ A et IgM observée chez le porcelet sevré (Corino et al 2002), la truie et les porcelets nouveau-nés (Bontempo et al 2004, Rossi et al 2004). Ces résultats sont en accord avec des données précédentes de Sugano et al (1998) chez la souris, qui ont mis en évidence une réduction des IgE. Dans le même temps, une réduction du TNF- $\alpha$ a été observée (Turek et al 1998). Un effet protecteur des CLA contre les défauts d'immunité a été observé par Cook et al (1993) chez le poulet. Hontecillas et al (2002) ont évalué les effets de la supplémentation en CLA sur l'inflammation de la muqueuse du côlon chez des porcelets, inflammation induite par une bactérie pathogène entérique (Brachyspira hyodysenteriae). La supplémentation du régime par des CLA avant l'induction de colite a diminué les effets négatifs au niveau de la muqueuse, maintenu le profil des cytokines (interferon- $\gamma$ et interleukin-10) et la distribution des sous-ensembles de lymphocytes (CD4 ${ }^{+}$ et $\mathrm{CD}^{+}$) ressemblait à celle de porcs non infectés. L'expression de PPAR- $\gamma$ au niveau du côlon a été augmentée.
Ainsi, des CLA introduits préventivement dans l'alimentation atténuent les lésions inflammatoires au début de la maladie entérique et en empêchent le développement ;

- une activité sur l'immunité non spécifique a été observée chez le porc, avec une augmentation du lysozyme (Bontempo et al 2004, Corino et al 2002) et une réduction des leucotriènes ;

- une activité anti-inflammatoire est aussi mise en évidence chez le porc avec une réduction des $\mathrm{PGE}_{2}$ (Whigham et al 2001). BassaganyaRiera et al (2001) ont observé chez le porc un effet de la supplémentation avec CLA sur les lymphocytes CD8 et les lymphocytes CD4.

L'apport des CLA chez la truie en fin de gestation induit une augmentation importante des IgG dans le colostrum (Bontempo et al 2004). Les IgG présents dans le colostrum dérivent essentiellement du sérum et leur captation par la glande mammaire est obtenue par des récepteurs de cellules épithéliales spécifiques (Huang et al 1992). Le taux d'IgG a été aussi significativement augmenté dans le sérum des truies traitées. En outre, le niveau des IgG dans le sérum des porcelets issus des mères traitées était significativement plus élevé que chez les porcelets non traités. L'immunité passive, dans la forme d'IgG absorbés à partir du colostrum et du lait, est vitale pendant les premiers jours de vie du porcelet car elle fournit pratiquement les seuls moyens de résister aux infections. Cependant le niveau des IgG dans le colostrum est fortement variable chez les truies recevant le même traitement pour différentes raisons. La dose initiale la plus élevée d'IgG du colostrum reçu par un porcelet dépend de l'ordre de naissance et la durée de la mise bas. Ceux nés le plus tardivement peuvent recevoir insuffisamment d'IgG du colostrum. En outre, une étude récente suggère que la synthèse IgG par des porcelets est directement à rapprocher de la quantité maternelle d'IgG reçue (Rooke et Bland 2002). Les niveaux d'IgG dans le sérum des porcelets en post-sevrage étaient plus élevés $(P<0,05)$ à j 25 et j 35 chez les animaux ayant reçu des CLA par rapport aux porcelets contrôles (figure 3). Corino et al (2002) ont aussi montré que la concentration d'IgG était plus élevée dans le sérum des porcelets alimentés à $0,5 \%$ et $1 \%$ de CLA pendant 28 jours après le sevrage que les témoins. La supplémentation en CLA a augmenté aussi la concentration des anticorps sériques contre Mycoplasma hyopneumoniae chez des porcelets sevrés (Weber et al 2001). Notre étude montre que l'apport alimentaire de CLA chez des porcelets en post-sevrage peut hâter le développement de l'immunité.

Le lysozyme, présent dans des sécrétions externes, polymorphonuclear leukocytes et macrophages, est fortement actif contre des bactéries Gram-positives. L'enzyme hydrolyse préférentiellement la liaison glycosidique $\beta-1,4$ entre l'acide $\mathrm{N}$-acétylmuramique et $\mathrm{N}$-acétylglucosamine présente dans la paroi cellulaire mucopeptidique de différents microorganismes. Pendant la lactation, nous avons constaté que les niveaux de lysozyme étaient plus élevés chez les truies et les porcelets rece-

Figure 3. Teneur en lgG du plasma des porcelets nés de truies alimentées avec rations supplémentées ou non avec CLA (CTR- ou CLA-) et nourris en post-sevrage avec ration supplémentée ou non avec CLA (-CLA ou -CTR).

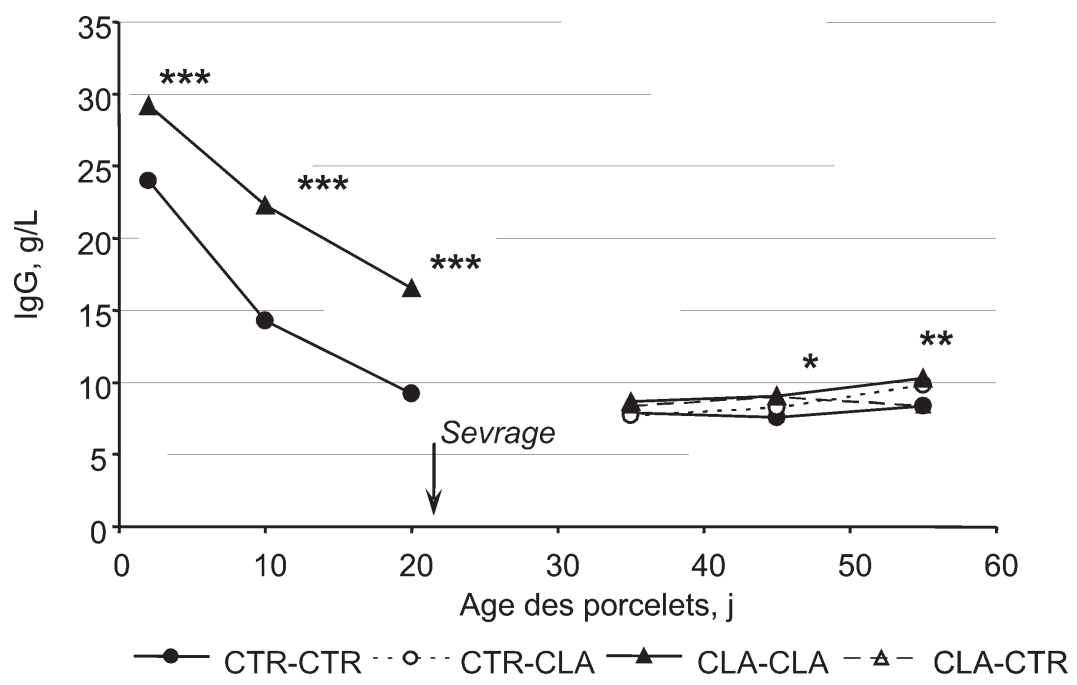


vant les CLA alimentaires que chez les témoins (Bontempo et al 2004). En post-sevrage les mêmes porcelets avaient des niveaux de lysozyme plus hauts $(\mathrm{P}<0,05)$ seulement à 25 jours. Ces données sont en accord avec celles de Corino et al (2002) chez des porcelets sevrés montrant que les CLA permettent d'augmenter le potentiel de défense antibactérien du corps.

Si l'influence des CLA sur la réponse immunitaire est maintenant confirmée par plusieurs études et chez différents modèles expérimentaux, il n'est pas encore possible de montrer l'influence des différents isomères pris séparément. Selon Yamasaki et al (2003, 2004) ils agiraient d'une façon différente et à différents niveaux. La forme d'administration des CLA, triglycérides ou acides gras libres, aurait également une influence vis-à-vis de la réponse immunitaire.

\section{4 / Conclusion}

Les CLA introduits dans l'alimentation du porc semblent influencer les performances de croissance grâce à une meilleure efficacité alimentaire et une diminution de l'adiposité de la carcasse.
Toutefois les effets n'apparaissent pas très marqués et semblent dépendre de la forme d'apport des CLA et du stade de distribution. La santé de l'animal serait améliorée et les CLA pourraient jouer un rôle important dans le futur en nutrition porcine pour leur action immunomodulatoire : ceci est très intéressant en particulier chez le porcelet nouveau né et en post sevrage pour répondre à la politique européenne de restriction dans l'utilisation des antibiotiques. Mais des études sont encore nécessaires pour montrer un réel intérêt en terme d'élevage.

La qualité technologique de la viande n'est pas affectée par l'incorporation des CLA dans l'alimentation, et il y aurait même un effet plutôt positif pour les critères de transformation de la viande. La qualité nutritionnelle de la viande semble améliorée en raison du dépôt des CLA dans la viande. Ceci est une piste particulièrement intéressante pour la mise en place de nouveaux produits si l'on estime que les CLA ont un rôle à jouer dans la santé publique visà-vis du développement de certaines tumeurs cancéreuses, de l'obésité et peut-être des maladies cardiovasculaires. En considérant que la consommation de CLA est maintenant comprise entre 100 et $500 \mathrm{mg}$ par jour (Ens et al 2001, Ritzenthalter et al 2001) et que les quantités proposées en nutrition humaine se situent autour des 3-4 g par jour (Gaullier et al 2002), il y a donc place pour développer des produits nouveaux enrichis en CLA. Toutefois, des études sont encore nécessaires pour identifier le CLA qui sera le plus efficace pour la santé humaine.

De plus, il existe un frein actuel au développement de cette incorporation de CLA dans l'alimentation des porcs qui est liée au coût d'utilisation du produit. Il faut donc envisager de développer de nouvelles techniques de production de CLA ou d'améliorer celles existantes afin de pouvoir mettre sur le marché ces acides gras à des prix acceptables pour la production porcine.

Mais avant d'envisager de généraliser l'utilisation des CLA en alimentation animale pour améliorer la valeur nutritionnelle de la viande, il faudra s'assurer de l'innocuité de tous les isomères des CLA vis-à-vis de la santé humaine ou bien utiliser des CLA débarrassés des isomères indésirables ce qui augmentera encore le coût de production.

\section{Références}

Azain M.J., 2003. Conjugated linoleic acid and its effects on animal products and health in single-stomached animals. Proc. Nutr. Soc., 62, 319-328.

Bassaganya-Riera J., Hontecillas R., Zimmerman D.R., Bregendahl K., Wannemuehler M. J., Zimmerman D.R., 2001. Effects of dietary conjugated linoleic acid in nursery pigs of dirty and clean environments on growth, empty body composition, and immune competence. J. Anim. Sci., 79, 714-721.

Bee G., 2000. Dietary conjugated linoleic acid consumption during pregnancy and lactation influences growth and tissue composition in weaned pigs. J. Nutr., 130, 2981-2989.

Bee G., 2001. Dietary conjugated linoleic acids affect tissue lipid composition but not de novo lipogenesis in finishing pigs. Anim. Res., 50, 383-399.

Bontempo V., Sciannimannico D., Pastorelli G., Rossi R., Rosi F., Corino C., 2004. Dietary conjugated linoleic acid positively affects immunologic variables in lactating sows and piglets. J. Nutr., 134, 817-824.

Chilliard Y., Ferlay A., Doreau M., 2001. Effect of different types of forages, animal fat or marine oils in cow's diet on milk fat secretion and composition, especially conjugated acid (CLA) and polyunsaturated fatty acids. Livest. Prod. Sci., 70, 31-48.
Chin S.F., Liu W., Storkson J.M., Ha Y.L., Pariza M.W., 1992. Dietary sources of conjugated dienoic isomers of linoleic acid, a newly recognized class of anticarcinogens. J. Food Comp. Anal., 5(3), 185-197.

Cook M.E., Miller C.C., Park Y., Pariza M.W. 1993. Immune modulation by altered nutrient metabolism: nutritional control of immuneinduced growth depression. Poultry Sci., 72, 1301-1305.

Corino C., Bontempo V., Sciannimanico D., 2002. Effects of dietary conjugated linoleic acid on some aspecific immune parameters and acute phase protein in weaned piglets. Can. J. Anim. Sci., 82, 115-117.

Corino C., Magni S., Pastorelli G., Rossi R., Mourot J., 2003. Effect of conjugated linoleic acid on meat quality, lipid metabolism, and sensory characteristics of dry-cured hams from heavy pigs. J. Anim. Sci., 81, 2219-2229.

Corino C., Filetti F., Gambarcorta M. Manchisi A., Magni S., Pastorelli G., Rossi R., Maiorano G., 2004. Influence of dietary conjugated linoleic acids (CLA) and age at slaughtering on meat quality and intramuscular collagen in rabbits. Meat Sci., 66, 97-103.

Corino C., Di Giancamillo A., Rossi R. Domeneghini C., 2005. Dietary conjugated linoleic acid affects morpho-functional and chemical aspects of subcutaneous adipose tissue in heavy pigs. J. Nutr., 135 (6), 1444-1450.
Demaree S.R., Gilbert C.D., Mersmann H.J., Smith S.B., 2002. Conjugated linoleic acid differentially modifies fatty acid composition in subcellular fractions of muscle and adipose tissue but not adiposity of postweanling pigs. J. Nutr., $132,3272-3279$.

Douard V., Etienne M., Père M.C., Mourot J., 2004a. Effet des Oméga 3 et des CLA alimentaires sur le développement du tissu adipeux chez le porc. 5émes Journ. Francophones de Nutrition, 13-15 décembre, Lyon, France, Nutrition Clinique et Métabolique, 18, suppl. 1, CO56.

Douard V., Mourot J., Mounier A., 2004b. Effet des CLA alimentaires sur les performances de croissance et la qualité de la viande de porc. 10 èmes Journ. Sciences du Muscle et Technologie des Viandes, 25/26 Octobre, Rennes, France, Viande et Produits Carnés, hors série, 41-42.

D'Souza D.N., Mullan B.P., 2002. The effect of genotype, sex and management strategy on the eating quality of pork. Meat Sci., 60, 95-101.

Dugan M.E.R., Aalhus J.L., Schaefer A.L., Kramer J.K.G., 1997. The effect of conjugated linoleic acid on fat to lean repartitioning and feed conversion in pigs. Can. J. Anim. Sci., 77, 723-725.

Dugan M.E.R., Aalhus J.L., Jeremiah L.E., Kramer J.K.G., Schaefer A.L., 1999. The effects of feeding conjugated linoleic acid on sub- 
sequent pork quality. Can. J. Anim. Sci., 79, 45-51.

Dugan M.E.R., Aalhus J.L., Lien K.A., Schaefer A.L., Kramer J.K.G., 2001. Effects of feeding different levels of conjugated linoleic acid and total oil to pigs on live animal performance and carcass composition. Can. J. Anim. Sci., 81, 505-510.

Dugan M.E.R., Aalhus J.L., Rolland D.C., Jeremiah L.E., 2003. Effects of feeding different levels of conjugated linoleic acid and total oil to pigs on subsequent pork quality and palatability. Can. J. Anim. Sci., 83, 713-720.

Dugan M.E.R., Aalhus J.L., Kramer J.K.G., 2004. Conjugated linoleic acid pork research. Am. J. Clin. Nutr., 79 (6), 2112S-2116S.

Dunshea F.R., Ostrowska E., Luxford B., Smits R.J., Campbell R.G., D'Souza D.N., Mullan B.P., 2002. Dietary conjugated linoleic acid can decrease backfat in pigs housed under commercial conditions. Asian Australasian J. Anim. Sci., 15, 1011-1017.

Eggert J.M., Belury M.A., Kempa-Steczko A., Mills S.E., Schinckel A.P., 2001. Effects of conjugated linoleic acid on the belly firmness and fatty acid composition of genetically lean pigs J. Anim. Sci., 79, 2866-2872.

Ens J.G., Ma D.W.L., Cole K.S., Field C.J., Clandinin M.T., 2001. An assessment of c9,t11 linoleic acid intake in a small group of young Canadians. Nutr. Res., 21, 955-960.

Gatlin L.A., See M.T., Larick D.K., Lin X., Odle J., 2002. Conjugated linoleic acid in combination with supplemental dietary fat alters pork fat quality. J. Anim. Sci., 80, 3105-3112.

Gaullier J., Berven G., Blankson H., Gudmunsen O., 2002. Clinical trial results support a preference for using CLA preparations enriched with two isomers rather than four isomers in human studies.Lipids, 37 (11), 10191025.

Gnadig S., Rickert R., Sebedio J.L., Steinhart H., 2001. Conjugated linoleic acid (CLA): physiological effects and production. Eur. J. Lipid Sci. Technol., 103, 56-61.

Hontecillas R., Wannemuehler M. J., Zimmerman D.R., Hutto D.L., Wilson J.H., Ahn D.U., Bassaganya-Riera J., 2002. Nutritional regulation of porcine bacterial-induced colitis by conjugated linoleic acid. J. Nutr., 132, 2019-2027.

Huang S.C., Hu Z.L., Hasler-Rapacz, J., Rapacz J., 1992. Preferential mammary storage and secretion of immunoglobulin gamma (IgG) subclasses in swine. J. Reprod Immunol., 42, 533-536.

Jahreis G., Kraft J., Tieschendorf F., Schone F., von Loeffelholz C., 2000. Conjugated linoleic acids: physiological effects in animal and man with special regard to body composition. Eur. J. Lipid Sci. Technol., 102, 695-703.

Joo S.T., Lee J.I., Ha Y.L., Park G.B., 2002. Effects of dietary conjugated linoleic acid on fatty acid composition, lipid oxidation, color, and water-holding capacity of pork loin. J. Anim. Sci., 80, 108-112.

Kang K.H., Albright K., Park Y., Pariza M.W. 2003. CLA inhibits differentiation of 3T3-L1 adipocytes and decreases PPAR gamma expression. Biochem. Biophys. Res. Comm., 303, 795799.

King D.A., Behrends J.M., Jenschke B.E., Rhoades R.D., Smith S.B., 2004. Positional dis- tribution of fatty acids in tryacilglycerols from subcutaneous adipose tissue of pigs fed diets enriched with conjugated linoleic acid, corn oil, or beef tallow. Meat Sci., 67, 675-681.

Kramer J.K.G., Cruz-Hernandez C., Deng Z.Y., Zhou J.Q., Jahreis G., Dugan M.E.R., 2004. Analysis of conjugated linoleic acid and trans 18:1 isomers in synthetic and animal products. Am. J. Clin. Nutr., Suppl. S.,79(6), 1137S$1145 \mathrm{~S}$.

Lebret B., Mourot J., 1998. Caractéristiques et qualité des tissus adipeux chez le porc. Facteurs de variation non génétiques. INRA Prod. Anim., 11, 131-143.

Lebret B., Lefaucheur L., Mourot J., 1999. Caractéristiques et qualité des tissus musculaires chez le porc. Facteurs de variation non génétiques. INRA Prod. Anim., 12, 11-28.

Lee K.N., Pariza M.W., Ntambi J.M., 1998. Conjugated linoleic acid decreases hepatic stearoyl- CoA desaturase mRNA expression. Biochem. Biophys. Res. Commun., 248, 817 821.

Martin J.C., Grégoire S., Siess M.H., Genty M., Chardigny J.M., Berdeaux O., Juaneda P., Sebedio J.L., 2000. Effects of conjugated linoleic acid isomers on lipid-metabolizing enzymes in male rats. Lipids, 35, 91-98.

Mersmann H.J., 2002. Mechanisms for conjugated linoleic acid-mediated reduction in fat deposition. J. Anim. Sci., 80 (suppl. 2), E126E134.

Migdal W., Pasciak K P., Wojtysiak D., Barowicz T., Pieska M., Pietras M., 2004. The effect of dietary CLA supplementation on meat and eating quality, and the histochemical profile of the m. longissimus dorsi from stress susceptible fatteners slaughtered at heavier weights. Meat Sci., 66, 863-870.

Mourot J., Hermier D. 2001. Lipids in monogastric animal meat. Reprod. Nut. Dev., 41, 109118.

Muller H.L., Kirchgessner L.M., Roth F.X., Stangl G.I., 2000. Effect of conjugated linoleic acid on energy metabolism in growing-finishing pigs. J. Anim. Physiol. Anim. Nutr., 83 (2), 8594

O’Quinn P.R., Nelssen J.L., Goodband R.D., Unruh J.A., Woodworth J.C., Smith J.S., Tokach M.D., 2000. Effects of modified tall oil versus a commercial source of conjugated linoleic acid increasing levels of modified tall oil on growth performance and carcass characteristics of growing-finishing pigs. J. Anim. Sci., 78, 23592368 .

O'Shea M., Bassaganya-Riera J., Mohede I.C.M., 2004. Immunomodulatory properties of conjugated linoleic acid. Am. J. Clin. Nutr., 79, 1199S-1206S.

Ostrowska M.E., Muralitharan R.F., Bauman D.E., Dunshea F.R., 1999. Dietary conjugated linoleic acids increase lean tissue and decrease fat deposition in growing pigs. J. Nutr., 129 , 2037-2042.

Ostrowska M.E., Cross R.F., Muralitharan R.F., Bauman D.E., Dunshea F.R., 2002. Effects of dietary fat and conjugated linoleic acid on plasma metabolite concentrations and metabolic responses to homeostatic signals in pigs. Br. J. Nutr., 88, 625-634.

Ostrowska M.E., Cross R.F., Muralitharan R.F., Bauman D.E., Dunshea F.R., 2003. Dietary conjugated linoleic acid differentially alters fatty acid composition and increases conjugated linoleic acid content in porcine adipose tissue. Br. J. Nutr., 90, 915-928.

Pariza, M.W., 2004. Perspective on the safety and effectiveness of conjugated linoleic acid Am. J. Clin. Nutr., 79, 1132s-1136s.

Park Y., Albright K.J., Liu W., Storkson J.M., Cook M.E., Pariza M.W., 1997. Effect of conjugated linoleic acid on body composition in mice. Lipids, 32, 853-858.

Ramsay T.G., Evock-Clover C.M., Steele N.C., Azain M.J., 2001. Dietary conjugated linoleic acid alters fatty acid composition of pig skeletal muscle and fat. J. Anim. Sci., 79, 21522161.

Ritzenthaler K.L., Mc Guire M.K., Falen R., Shultz T.D., Dasgupta N., Mc Guire M.A., 2001. Estimation of conjugated linoleic acid intake by written dietary assessment methodologies under estimates actual intake evaluated by food duplicate methodology. J. Nutr., 131, 1548-1554.

Rooke J.A., Bland I.M., 2002. The acquisition of passive immunity in the new-born piglet. Livest. Prod. Sci., 78, 13-23.

Rossi R, Pastorelli G, Bontempo V, Corino C. 2004. Effects of dietary conjugated linoleic acid (CLA) on immunoglobulin concentration in sow colostrum and piglet serum. Vet. Res. Commun., Sp. Iss. SI Suppl. 1, 28(1), 241-244.

Smith S.B., Hively T.S., Cortese G.M., Han J.J., Chung K.Y., Castenada P., Gilbert C.D., Adams V.L., Mersmann H.J., 2002. Conjugated linoleic acid depresses the ? 9 desaturase index and stearoyl coenzyme A desaturase enzyme activity in porcine subcutaneous adipose tissue. J. Anim. Sci., 80, 2110-2115.

Sugano M., Tsujita A., Yamasaki M., Noguchi M., Yamada K., 1998. Conjugated linoleic acid modulates tissue levels of chemical mediators and immunoglobulins in rats. Lipids, 33, 521527.

Thiel-Cooper R.L., Parrish F.C., Sparks J.C., Wiegand B.R., Ewan R.C., 2001. Conjugated linoleic acid changes swine performance and carcass composition. J. Anim. Sci., 79, 18211828.

Tischendorf F., Shone F., Kircheim U., Jahreis G., 2002. Influence of a conjugated linoleic acid mixture on growth, organ weights, carcass traits and meat quality in growing pigs. J. Anim. Physiol. Anim. Nutr., 86, 117-128.

Turek J.J., Li Y., Schoenlein I.A., Allen K.G.D., Watkins B.A., 1998. Modulation of macrophage cytokine production by conjugated linoleic acids is influenced by the dietary $n-6-n-$ 3 fatty acid ratio. J. Nutr. Biochem., 9, 258-266.

Wang G Y., Jones P.J.H., 2004. Dietary conjugated linoleic acid and body composition. Am. J. Clin. Nutr., 79, 1153S-1158S.

Waylan A.T., O'Quinn P.R., Unruh J.A. Nelsen J.L., Goodband R.D., Woodworth J.C., Tokach M.D., Koo S.I., 2002. Effects of modified tall oil and vitamin E on growth performance, carcass characteristics, and meat quality of growing-finishing pigs. J. Anim. Sci., 80, 1575 1585

Weber T.E., Schinkel A.P., Hoseknecht K.L. Richert B.T., 2001. Evaluation of conjugated linoleic acid and dietary antibiotics as growth promotants in weanling pigs. J. Anim. Sci., 79, 2542-2549.

West D.B., Delany J.P., Camet P.M., Blohm F., Truett A.A., and Scimeca J., 1998. Effects of conjugated linoleic acid on body fat and energy 
metabolism in the mouse. Am. J. Physiol., 44, R667-R672.

West D.B., Blohm F., Truett A.A., Delany J.P., 2000. Conjugated linoleic acid persistently increases total energy expenditure in $\mathrm{AKR} / \mathrm{J}$ mice without increasing uncoupling protein gene expression. J. Nutr., 130 (10), 2471-2477.

Whigam L.D., Cook E.B., Stahl J.L., Saban R., Bjorling D.E., Pariza M.W., Cook M.E., 2001. CLA reduces antigen-induced histamine and PGE(2) release from sensitized guinea pig tracheae. Am. J. Physiol. Reg., 280, R908-R912.

Wiegand B.R., Parrish F.C. Jr, Swan J.E., Larsen S.T., Baas T.J., 2001. Conjugated linoeic acid improves feed efficiency, decreases subcutaneous fat, and improves certain aspects of meat quality in Stress-Genotype pigs. J. Anim. Sci. 79, 2187-2195.

Wiegand B.R., Sparks J.C., Parrish F.C., Zimmerman D.R., 2002. Duration of feeding conjugated linoleic acid influence growth performance, carcass traits, and meat quality of finishing barrows. J. Anim. Sci., 80, 637-643.

Wood J.P., Enser M.B., Mac Fie H.J.H., Smith W.C., Chadwick J.P., Ellis M., Laird R., 1978. Fatty acid composition of backfat in Large White pigs selected for low backfat thickness. Meat Sci., 2, 289-296.

Yamasaki M., Chujo H., Hirao A., Koyanag N., Okamoto T., Tojo N., Oishi A., Iawata T., Yamauchi-Sato Y., Yamamoto T., Tsutsumi K., Tachibana H., Yamada K., 2003. Immuno- globulin and cytokine production from spleen lymphocytes is modulated in $\mathrm{C} 57 \mathrm{BL} / 6 \mathrm{~J}$ mice by dietary cis-9, trans -11 and trans -10 , cis-12 conjugated linoleic acid. J. Nutr., 133, 784-788.

Yamasaki M, Kitagawa T, Chujo H, Koyanagi N, Nishida E, Nakaya M, Yoshimi K, Maeda H, Nou S, Iwata T, Ogita K, Tachibana H, Yamada K., 2004. Physiological difference between free and triglyceride-type conjugated linoleic acid on the immune function of $\mathrm{C} 57 \mathrm{BL} / 6 \mathrm{~N}$ mice. $\mathrm{J}$. Agric. Food Chem., 52(11), 3644-3648.

Yu L., Adams, D., Watkins, B.A., 2003. Comparison of commercial supplements containing conjugated linoleic acid. J. Food Composition Anal., 16, 419-428.

\section{Résumé}

Les CLA ont plusieurs effets biologiques intéressants vis-à-vis de la santé : ils ont une propriété anticarcinogène, une activité anti-athérosclérose et antioxydante. Ils diminuent l'obésité et apparaissent comme un facteur capable de stimuler la réponse immunitaire.

En nutrition porcine, l'influence sur les performances de croissance est limitée et on peut observer seulement un faible effet positif sur l'efficacité alimentaire. La composition corporelle et les paramètres de la qualité de la viande sont peu modifiés, l'adiposité apparaissant diminuée avec les CLA. La composition en acides gras (AG) du tissu adipeux et du muscle est modifiée par le traitement avec CLA : la teneur en AG saturés est augmentée et la teneur en AG monoinsaturés est diminuée. Les CLA sont retrouvés dans le muscle et dans le tissu adipeux des porcs en ayant ingéré. La quantité déposée dépend de la consommation journalière.

Les CLA ont un important effet immunomodulatoire. Chez la truie alimentée avec un régime contenant des CLA, on peut observer dans le colostrum une concentration très élevée en IgG, IgM et IgA avec un effet positif sur l'immunité passive des porcelets et sur leur croissance.

L'influence des CLA sur la composition en lipides du tissu adipeux peut être très importante d'un point de vue technologique pour le contenu plus élevé en acides gras saturés et d'un point de vue nutritionnel en raison de l'apport de CLA. De même l'effet immuno-modulatoire chez la truie pourra permettre de jouer un rôle intéressant vis-à-vis de l'immunité chez le porcelet.

\section{Abstract}

\section{Conjugated linoleic acid in pig nutrition}

The interest in CLA is centred on several biological properties that relate to health: anticarcinogenic, anti-obesity, anti-atherogenic and immunomodulatory functions. The amount of literature on CLA is growing at a phenomenal rate and today an evaluation of the influence of conjugated linoleic acid (CLA) on growth, carcass characteristics, meat quality and immune response in pigs is possible.

No significant differences were observed on average daily gain, feed intake and a tendency for higher feed efficiency is reported. Limited effects on carcass characteristics and meat quality were observed. Backfat thickness was reduced in CLA fed pigs. Fatty acid composition of ham fat was significantly affected by dietary CLA. Higher saturated fatty acids, lower monounsaturated fatty acids and higher CLA content were observed in fat of CLA fed pigs.

Dietary CLA had a positive effect on immune parameters of lactating sows and piglets. Feeding CLA increased sow colostrum IgG, IgA and IgM. Nursing piglets from CLA-fed sows had significantly higher serum lysozyme and IgG.

These data suggest that conjugated linoleic acid have no, or limited, effects on growth performances, carcass characteristics and meat quality of pigs. The influence of CLA on fatty acid composition of adipose tissue may be very important from a technological point of view for the higher content of saturated fatty acids and from a nutritional point of view for the higher CLA content. CLA can affect cellular and humoral responses to antigen challenge, affecting both adaptive and innate responses in pigs.

CORINO C., PASTORELLI G., DOUARD V., ROSSI R., MUSELLA M., MOUROT J., 2006. L'acide linoléique conjugué en nutrition porcine. INRA Prod. Anim., 19, 39-46. 